\title{
Design of Unmanned Wing-in-ground Effect Vehicle with Tri-fold Main Wing
}

\author{
Xuecheng Shuang ${ }^{1}$, Yan Zhang ${ }^{1 *}$ \\ ${ }^{1}$ Shenlan technology Innovation Team, School of Energy and Power Engineering, Wuhan University of Technology (WUT), Wuhan, \\ Hubei Province, 430063, China
}

\begin{abstract}
This paper proposed an innovative design of unmanned wing-in-ground effect vehicle with tri-fold main wing, aiming at optimizing the main wing of unmanned vehicles based on its current application. The design also takes into account the speed and load in an effort to improve efficiency and broaden the application of the vehicle. In addition, the unmanned GEV is driven by electric power and propelled by the main and auxiliary propellers, which is well supported by the ground effect. The design features energy conservation and emission reduction, which is worthy of promoting in the market.
\end{abstract}

\section{Background}

Nowadays, unmanned vehicles have greatly facilitated traditional manual navigation, while there are also drawbacks in its application. For unmanned work like patrol, monitoring, investigation and mapping in large areas of water, it is difficult for the existing unmanned vehicles to feature both efficiencies brought by speed and multi-functional performance supported by heavy load capacity. The high-performance unmanned vehicle, or unmanned GEV, has been developing rapidly in recent years [1], which has partially solved the above problems. However, the design of the main wing has reduced the mobility of the hull when sailing in water.

Therefore, the paper proposed an innovative design of unmanned GEV with tri-fold main wing that features both speed and load capacity targeting defects in the existing GEV, thus improving the efficiency of operation and energy conservation, broadening its application, and reducing emission.

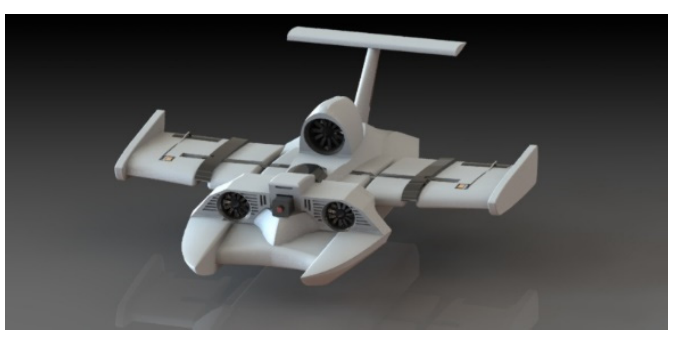

\section{Principle of Design}

\subsection{Overall structural design}

The unmanned GEV with a tri-fold main wing structure (unmanned GEV for short) in this paper consists of a biplate hull, wings and propellers. The length of the hull is about $900 \mathrm{~mm}$, and the theoretical speed is $60-70 \mathrm{~km} / \mathrm{h}$ when sailing with ground effect and $20-40 \mathrm{~km} / \mathrm{h}$ when sailing under water drainage.

\subsection{Structural design of the folded main wing}

The structure of the tri-fold main wing in this paper is shown in Figure 1. In this structure, the movement of the three connecting rod is driven by the expansion and contraction of the hydraulic rod, thus allowing folding of the structure. When the main wing is unfolded, the three connecting rod serves as an auxiliary structure that supports the main wing to be folded towards the hull. The structures of the three connecting rod and the hydraulic rod are shown in Figure 2.

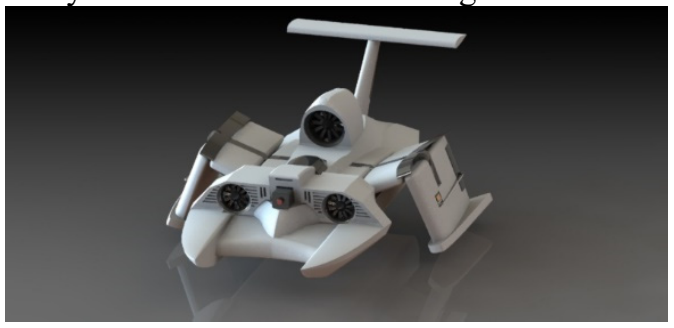

Figure 1. Sketch of Tri-fold Main Wing. 

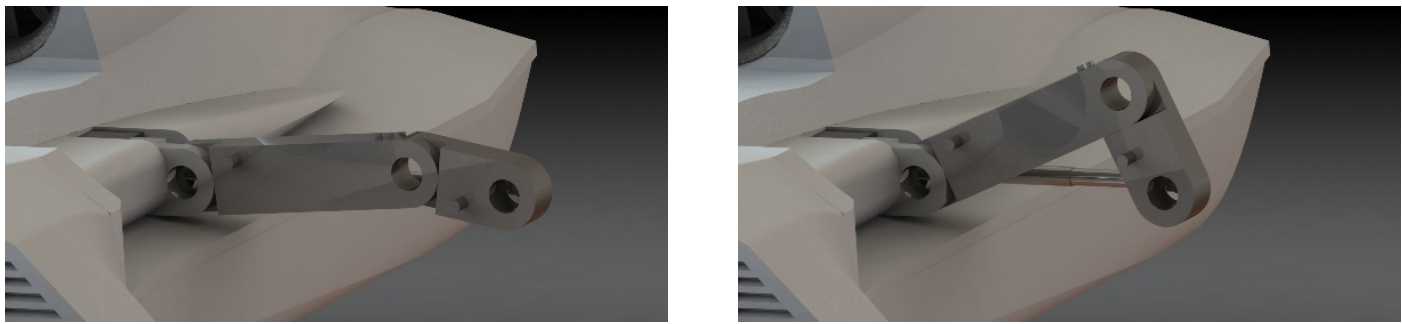

Figure 2. Diagram of Three Connecting Rod and Hydraulic Piston Rod (Screw Omitted).

\subsection{Design of propellers}

The unmanned GEV is equipped with two $50 \mathrm{~mm}$ ducted fans with the power of $P_{1}=670 \mathrm{w}$ as auxiliary propellers and a $64 \mathrm{~mm}$ ducted fan with the power of $P_{2}=874 \mathrm{w}$ as the main propeller, as shown in Figure 3. Driven by lithium battery power, the unmanned GEV makes a full use of the ground effect when sailing at high speed while the auxiliary propeller stays inactive, which is conducive to the maintenance of speed and reduction of energy consumption, thus promoting energy conservation and emission reduction.

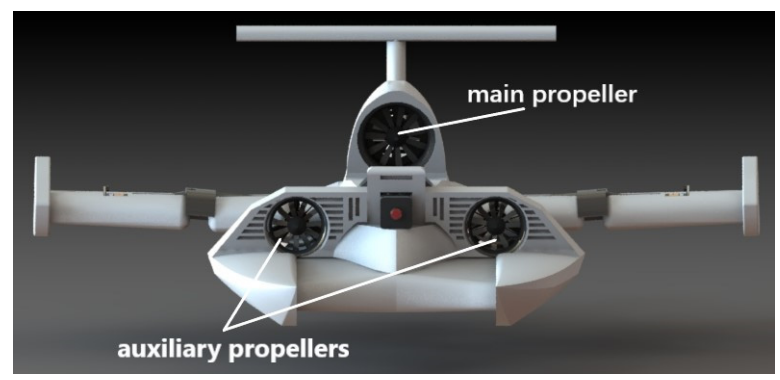

\section{Verification Analysis}

\subsection{Overall strength analysis}

Expanded polypropylene (EPP) was used in the hull of unmanned GEV, which is a new eco-friendly compression-resistant, buffering and heat-insulating material that can be recycled and degraded without causing pollution. A three-dimensional model was established by SolidWorks, where the overall strength of the unmanned GEV was simulated and analyzed using ANSYS WORKBENCH. The maximum external force on the weakest part of the unmanned GEV was adopted. That is, the deformation of the front part of the hull under the maximum external impact when landing without the ground effect, as shown in Figure 4. According to the simulation results, the maximum deformation was $0.024991 \mathrm{~mm}$. As the EPP is an extremely versatile plastic foam, the deformation meets the requirement for use.

Figure 3. Structure of Main and Auxiliary Propeller.

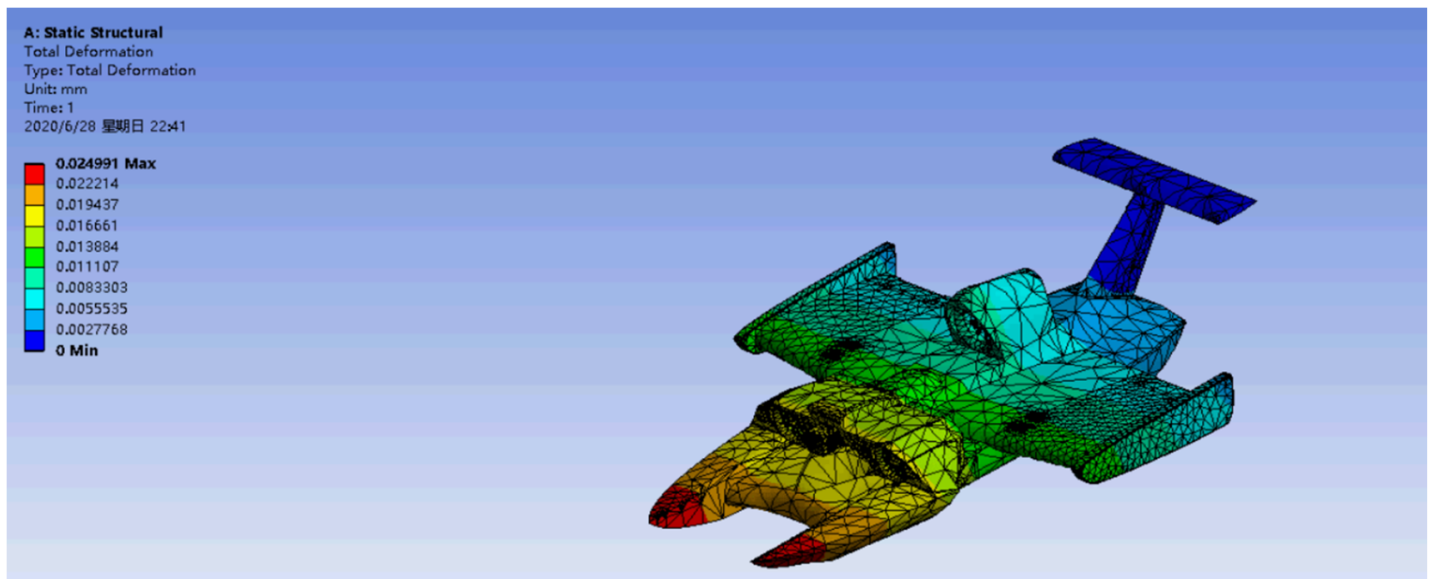

Figure 4. Simulation of Overall Strength.

Table 1. The Width of Main Wing.

\subsection{Benefit analysis of main wing folding}

3.2.1. Improved mobility. When the unmanned GEV navigates in water with a tri-fold main wing, the large-scale reduction in the width of the vehicle would greatly improve its mobility. The width of the main wing before and after folding is shown in Table 1.

\begin{tabular}{ccc}
\hline $\begin{array}{c}\text { The Width of } \\
\text { Main Wing } \\
\text { before Folding } \\
(\mathrm{mm})\end{array}$ & $\begin{array}{c}\text { The Width of } \\
\text { Main Wing after } \\
\text { Folding (mm) }\end{array}$ & $\begin{array}{c}\text { Scale of } \\
\text { Reduction }\end{array}$ \\
\hline 746.3 & 527.8 & $29.3 \%$ \\
\hline
\end{tabular}


3.2.2. Rapid landing. When the unmanned GEV lands on the water surface without the ground effect, the tri-fold structure of the main wing allows rapid landing. This paper, based on existing studies of folded main wing[2], simulated the angle of folding, lift coefficient $C_{L}$ and drag coefficient $C_{D}$ of the GEV using DATCOM and MATLAB[3]. The results showed that:

$$
\begin{aligned}
& L=Q \cdot S_{W} \cdot C_{L} \\
& D=Q \cdot S_{W} \cdot C_{D}
\end{aligned}
$$

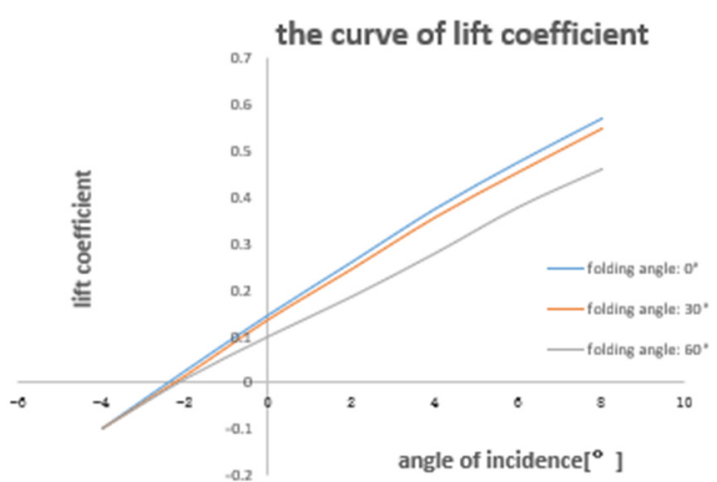

Figure 5. Curve of $C_{L}$.
$\mathrm{L}$ and $\mathrm{D}$ represent the lift force and resistance generated by aerodynamic force. $Q$ is the dynamic pressure, $Q=0.5 \cdot \rho \cdot V^{2} . \rho$ is the air density, and $V$ the air flow velocity (i.e. sailing velocity). $S_{W}$ is the reference area of the main wing. Based on the formula, the curve of $C_{L}$ and angle of incidence $\alpha$, the curve of $C_{D}$ and angle of incidence $\alpha$, and the curve of lift versus drag coefficient (also referred to as $C_{L} / C_{D}$ ) at the folding angle of $0^{\circ}, 30^{\circ}$ and $60^{\circ}$ are shown in Figure 5, 6 and 7 respectively.

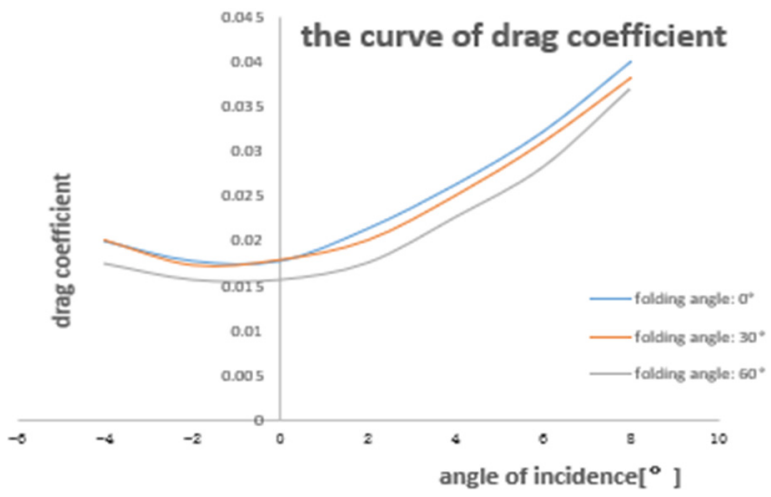

Figure 6. Curve of $C_{D}$.

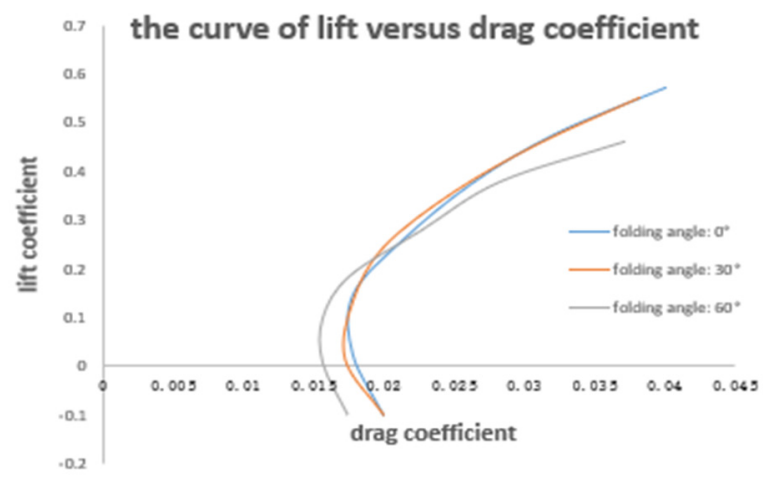

Figure 7. Curve of $C_{L} / C_{D}$ Ratio.

Based on analysis of the $C_{L} / C_{D}$ curve, it was concluded that when the GEV lands at the same angle of incidence, it will experience decreasing lift and drag coefficient as well as lower $C_{L} / C_{D}$ ratio, thus allowing rapid landing.

3.2.3. Increased cushion and lateral stability. When rapid landing is available for unmanned GEV without ground effect via the tri-fold structure of the main wing, the folded main wing also plays a buffering role in landing and disperses impact from the water surface when landing. The model of Stress Analysis of the Tri-fold Main Win and stress distribution are shown in Figure 8 and Figure 9. 


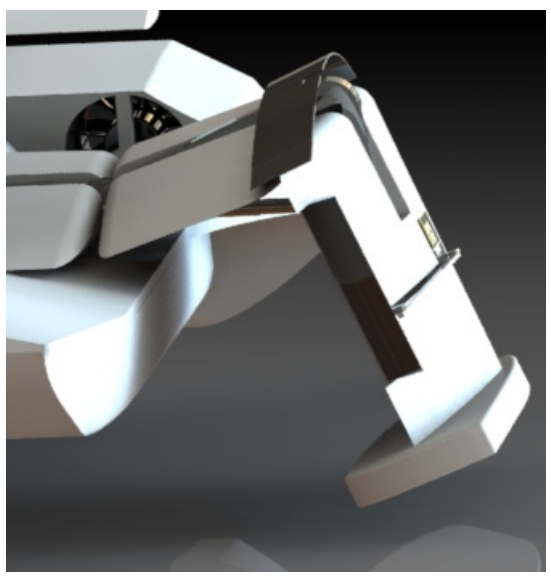

Figure 8. Model of Stress Analysis of the Tri-fold Main Wing.

Stress analysis using ANSYS WORKBENCH found that the maximum stress of the main wing was $0.1313 \mathrm{MPa}$, within the capacity of both hydraulic rods (45 steel) and the three connecting rods (45 steel). Meanwhile, the folded main wings are distributed on both sides of the hull in contact with the water surface when sailing, thus increasing the lateral stability of the hull.

\subsection{Analysis of the lift force of the wing}

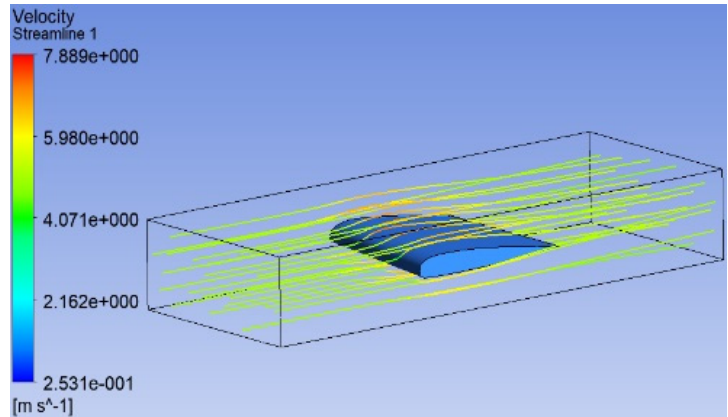

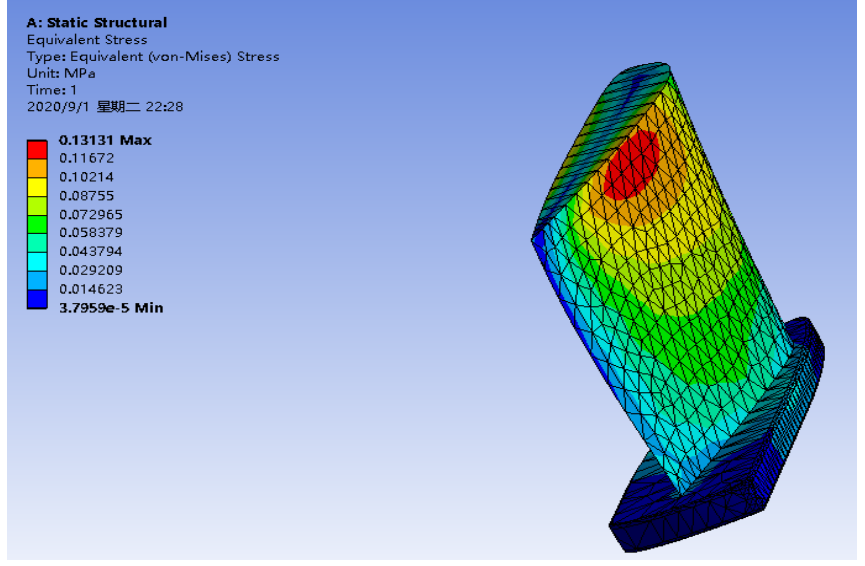

Figure 9. Stress Distribution.

A pair of main wings and horizontal tail wing are responsible for the lift of the unmanned GEV. The simplified model of single main wing and horizontal tail wing of unmanned GEV using ANSYS WORKBENCH is shown in Figure 10. Lift simulation analysis was carried out, where the fixed wing was adopted, and the flow velocity was set at $60 \mathrm{~km} / \mathrm{h}$ to simulate the navigation of unmanned GEV at the lowest ground-effect speed. In this situation, the lift force generated by the wing at a stable level served as the basis of analysis, as shown in Figure 11.

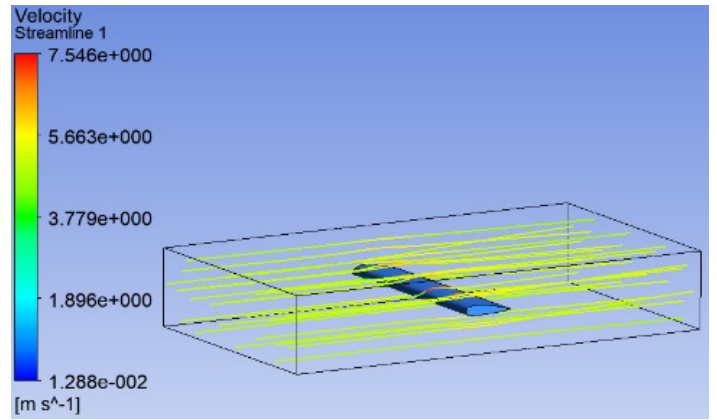

Figure 10. Simplified Model of Main Wing and Horizontal Tail Wing.

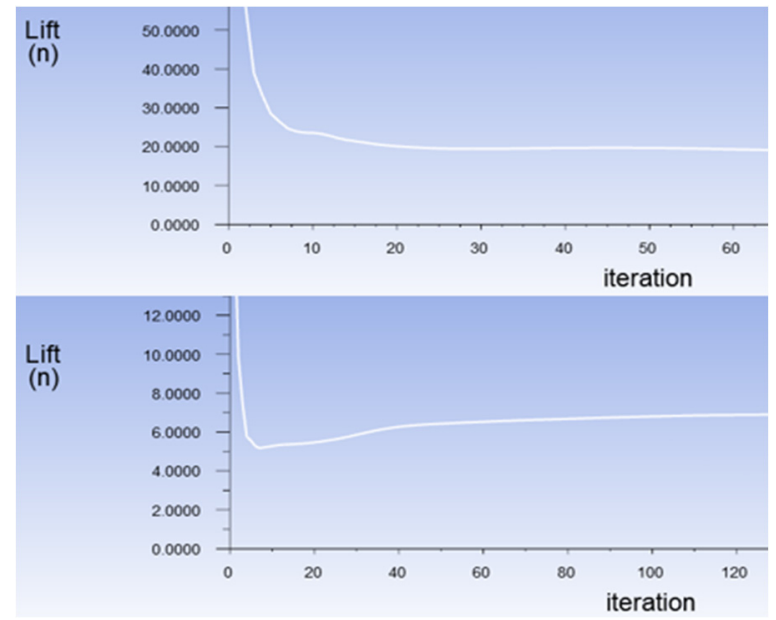

The ratio of the total lift generated by the wing of the unmanned GEV to the gravity was calculated to approximate 0.96. Combined with the ratio of thrust and gravity of the unmanned GEV (or the thrust-weight ratio), the value was approximating 0.9 , allowing navigation with ground effect.

\subsection{Analysis of energy consumption caused by switch of propulsion modes}

The unmanned GEV in this paper was driven by lithium battery power, and was equipped with two $50 \mathrm{~mm}$ ducted fans with the power of $P_{1}=670 \mathrm{w}$ as auxiliary propellers and one $64 \mathrm{~mm}$ ducted fan with the power of $P_{2}=874 \mathrm{w}$ as the main propeller. The unmanned GEV would make a full use of the ground effect when sailing at high speed while the auxiliary propeller stays inactive, with the main propeller as the driver of high-speed navigation. Compared with energy consumption of 
full-load operation during underwater navigation, this type of sailing features much lower energy consumption:

$$
\eta=2 \cdot P_{1} \div\left(2 \cdot P_{1}+P_{2}\right) \approx 60.52 \%
$$

In this way, the vehicle is allowed to sail at a high speed while greatly reducing energy consumption, which is conducive to energy conservation and emission reduction.

\section{Conclusion}

This paper proposed an innovative design of unmanned wing-in-ground effect vehicle with tri-fold main wing, aiming at optimizing the main wing of unmanned vehicles based on its current application. The design also takes into account the speed and load in an effort to improve efficiency and broaden the application of the vehicle. In addition, the unmanned GEV is driven by electric power and propelled by the main and auxiliary propellers, which is well supported by the ground effect. The design features energy conservation and emission reduction, which is worthy of promoting in the market.

\section{Acknowledgments}

This paper was support by Wuhan University of Technology National innovation and entrepreneurship training program for college students (Item Number: S202010497151) and Wuhan University of Technology innovation and entrepreneurship training program for college students (Item Number: 206805019).

\section{References}

1. Guo, P.F., Zhang Y., Shao Q.H. (2014) Study on the Development of WIG Wings in the World. Ship Standardization Engineer, 2: 49-50.

2. Ahmed, M.R., Sharm, S.D. (2005) An investigation on the aerodynamics of a symmetrical airfoil in ground effect. Experimental Thermal and Fluid Science, 29: 633-647.

3. Xue, C. (2018) An Investigation on Flight Control of Folded-wing Aircraft during Deformation. Nanjing University of Aeronautics and Astronautics,: 13-18. 